\title{
AN ALGEBRAIC ANALOGUE OF A CONJECTURE OF G. W. WHITEHEAD
}

\author{
HAYNES MILLER ${ }^{1}$
}

\begin{abstract}
Using a theorem of W. M. Singer, an algebraic analogue of a conjecture of G. W. Whitehead is proved, generalizing the algebraic Kahn-Priddy theorem of W. H. Lin.
\end{abstract}

Let $\operatorname{Sp}^{n}(X)$ denote the symmetric product $X^{n} / \Sigma_{n}$ of a space $X$. If $X$ is pointed we have a natural map $\operatorname{Sp}^{n}(X) \rightarrow \operatorname{Sp}^{n+1}(X)$ obtained by introducing the base point as the last coordinate. Dold and Thom [3] showed that the limit space $\operatorname{Sp}^{\infty}(X)$ is an Eilenberg-Mac Lane space with homotopy $\bar{H}_{*}(X ; \mathbf{Z})$, and that $X \rightarrow \operatorname{Sp}^{\infty}(X)$ induces the Hurewicz homomorphism in homotopy. In [8], G. W. Whitehead conjectured that in the stable range localized at a prime $p$,

$$
\operatorname{ker}\left(\pi_{*} \operatorname{Sp}^{p^{n}} X \rightarrow \pi_{*} \operatorname{Sp}^{p^{n+1}} X\right)=\operatorname{ker}\left(\pi_{*} \operatorname{SP}^{p^{n}} X \rightarrow \pi_{*} \operatorname{Sp}^{\infty} X\right) .
$$

In contemplating this conjecture, it should be borne in mind that if the interval $(i, j]$ does not contain a power of $p$, then, in the stable range localized at $p$, $\mathrm{Sp}^{i} X \rightarrow \mathrm{Sp}^{j} X$ is a homotopy equivalence [11].

Whitehead's conjecture turned out to have been too optimistic; in his thesis [13] P. J. Welcher produced a counterexample, which is very likely typical, for $X=S^{n}$ $\cup_{\nu} e^{n+4}$. The case of greatest interest is that of $X$ a sphere, however, and this remains open.

Given a spectrum $X$ one may form a spectrum $\operatorname{Sp}^{n}(X)$ in an obvious way; if $X$ is connective, the "stable range" restrictions then care for themselves. $\operatorname{Sp}^{\infty}\left(S^{0}\right)$ is the integral Eilenberg-Mac Lane spectrum $H$. Henceforth all spectra will be implicitly localized at 2. One may form the sequence of projections

$$
H \rightarrow H / S^{0} \rightarrow H / \mathrm{Sp}^{2} S^{0} \rightarrow \cdots \rightarrow H / \mathrm{Sp}^{2^{n}} S^{0} \rightarrow \cdots,
$$

and for the sphere Whitehead's conjecture (at the prime 2) amounts to the claim that each map is trivial in homotopy.

A convenient reformulation of this conjecture may be obtained by splicing together the resulting short exact sequences

$$
0 \rightarrow \pi_{*}\left(H / \mathrm{Sp}^{2^{n}} S^{0}\right) \rightarrow \pi_{*}\left(\mathrm{Sp}^{2^{n}} S^{0} / \mathrm{Sp}^{2^{n-1}} S^{0}\right) \rightarrow \pi_{*}\left(H / \mathrm{Sp}^{2^{n-1}} S^{0}\right) \rightarrow 0
$$

to obtain a long exact sequence

$$
0 \leftarrow \mathbf{Z}_{(2)} \stackrel{\varepsilon}{\leftarrow} \pi_{*}\left(S^{0}\right) \stackrel{\partial}{\leftarrow} \pi_{*}\left(\mathrm{Sp}^{2} S^{0} / S^{0}\right) \stackrel{\partial}{\leftarrow} \pi_{*}\left(\mathrm{Sp}^{4} S^{0} / \mathrm{Sp}^{2} S^{0}\right) \stackrel{\partial}{\leftarrow} \cdots
$$

Received by the editors December 1, 1980.

1980 Mathematics Subject Classification. Primary 55T15, 55S15, 55 S10.

${ }^{1}$ Supported in part by NSF grant MCS8002780. 
in which $\partial$ has degree -1 . An easy inductive diagram chase shows that conversely (0.2) implies Whitehead's conjecture as originally formulated.

The sequence $(0.2)$ is clearly exact at $\mathbf{Z}_{(2)}$. For the next step, recall that James, Thomas, Toda and Whitehead [4] showed that $\operatorname{Sp}^{2} S^{0} / S^{0} \simeq \Sigma \mathbf{R} P^{\infty}$ and that the resulting map $\mathbf{R} P^{\infty} \rightarrow S^{0}$ is induced by the $J$-homomorphism. Kahn and Priddy [5] showed that this map is onto in positive stems, verifying exactness at $\pi_{*}\left(S^{0}\right)$.

The symmetric product filtration of the integral Eilenberg-Mac Lane spectrum $H \simeq \mathrm{Sp}^{\infty} S^{0}$ has a nice description in cohomology. To state it, let $A$ be the Steenrod algebra and let $\bar{A}=H^{*}(H)=A / A \mathrm{Sq}^{1}$. Then $\bar{A}$ has a basis given by admissible squares not ending in $\mathrm{Sq}^{1}$. Let $\bar{F}^{n}$ be the sub- $A$-module with vector-space basis given by such squares of length at least $n$. Then [11] $0 \rightarrow \bar{F}^{n} \rightarrow \bar{A} \rightarrow H^{*}\left(\mathrm{Sp}^{2^{n-1}} S^{9}\right)$ $\rightarrow 0$ is exact. The algebraic analogue of Whitehead's conjecture (for $X=S^{0}$ ) is then our main result here:

MAIN TheOrem. $\bar{F}^{n+1} \rightarrow \bar{F}^{n}$ induces the trivial map in $\operatorname{Ext}_{A}^{*}\left(-, \mathbf{Z}_{2}\right)$ for $n \geqslant 0$.

As before, this is equivalent to the exactness of the sequence

$$
\begin{aligned}
0 \leftarrow \mathbf{Z}_{2}\left[h_{0}\right] \stackrel{\varepsilon}{\leftarrow} \operatorname{Ext}_{A}^{*}\left(\mathbf{Z}_{2}, \mathbf{Z}_{2}\right) \stackrel{\delta}{\leftarrow} \operatorname{Ext}_{A}^{*}\left(\bar{H}^{*} \mathbf{R} P^{\infty}, \mathbf{Z}_{2}\right) \\
\stackrel{\delta}{\leftarrow} \operatorname{Ext}_{A}^{*}\left(\bar{F}^{2} / \bar{F}^{3}, \mathbf{Z}_{2}\right) \leftarrow \cdots,
\end{aligned}
$$

where we have used the isomorphisms $\bar{F}^{0} / \bar{F}^{1}=\mathbf{Z}_{2}, \bar{F}^{1} / \bar{F}^{2}=\bar{H}^{*} \mathbf{R} P^{\infty}$, and $\operatorname{Ext}_{A}^{*}\left(\bar{A}, \mathbf{Z}_{2}\right)=\mathbf{Z}_{2}\left[h_{0}\right],\left|h_{0}\right|=(1,1)$. Once again, exactness at $\mathbf{Z}_{2}\left[h_{0}\right]$ is trivial. Exactness at the next stage is equivalent to the "algebraic Kahn-Priddy theorem" of W. H. Lin [7].

This long exact sequence is interesting from several points of view. Each $\delta$ raises homological degree by 1 , so, for given $s, \operatorname{Ext}_{A}^{s}\left(\mathbf{Z}_{2}, \mathbf{Z}_{2}\right)$ sits in a finite exact sequence. On the other hand, Welcher [14] proved that $\bar{F}^{n} / \bar{F}^{n+1}$ is $A(n-1)$-free, if $A(n-1)$ is the subalgebra of $A$ generated by $\mathrm{Sq}^{i}, i \leqslant 2^{n-1}$. By the theorem of Anderson and Davis [2], [10], therefore,

$$
\operatorname{Ext}_{A}^{s, t}\left(\bar{F}^{n} / \bar{F}^{n+1}, \mathbf{Z}_{2}\right)=0 \text { for } t-s+c_{n}<\left(2^{n+1}-2^{[n / 2]}-1\right) s
$$

where $c_{n}$ is a suitable constant. Thus the terms in the exact sequence have vanishing lines of progressively lower slope.

In [12], W. M. Singer proved a slightly weakened form of Lin's Kahn-Priddy theorem by a much simpler route. In $\$ 2$ of this note, we use Singer's method to prove an analogous weakening (Theorem 2.2) of the Main Theorem, and then in $\$ 3$ show that it actually implies the result as stated. We also give (in $\$ 1$ ) a derivation of the elementary properties of Singer's construction which is simpler than his original approach.

I am grateful to Singer for letting me see early versions of [12], and to N. J. Kuhn for providing the proof of Lemma 1.3.

1. Let $S$ denote the free associative $\mathbf{Z}_{2}$-algebra generated by $\left\{\mathrm{Sq}^{i}: i>1\right\}$, $\left|\mathrm{Sq}^{i}\right|=i$, with Hopf algebra structure determined by

$$
\Delta \mathrm{Sq}^{n}=\sum_{i+j=n} \mathrm{Sq}^{i} \otimes \mathrm{Sq}^{j}, \quad \mathrm{Sq}^{0}=1
$$


The Steenrod algebra $A$ is a quotient Hopf algebra of $S$. Let $P$ denote the vector space $\mathbf{Z}_{2}\left[x, x^{-1}\right],|x|=1$. Following W. M. Singer, we define a functor $\mathcal{P}$ from the category of $S$-modules to itself by setting $\mathscr{P}(M)=P \otimes M$ with $S$-action

$$
\mathrm{Sq}^{k}\left(x^{l-1} \otimes m\right)=\sum_{j}\left(\begin{array}{c}
l-j-1 \\
k-2 j
\end{array}\right) x^{k+l-j-1} \otimes \mathrm{Sq}^{j} m .
$$

The binomial coefficient $\left(\begin{array}{c}a \\ k\end{array}\right)$ here and throughout this paper is defined for all $a \in \mathbf{Z}$ and $k \in \mathbf{N}=\{0,1,2, \ldots\}$ by $(1+t)^{a}=\Sigma_{k}\left(\begin{array}{l}a \\ k\end{array}\right) t^{k}$.

The vector space $P$ is of course an $A$-module (and hence an $S$-module) via $\mathrm{Sq}^{k} x^{l-1}=\left(\begin{array}{c}l-1 \\ k\end{array}\right) x^{k+l-1}$, and one may thus form the diagonal tensor product $S$ module $P \otimes^{\Delta} M$. This is in general distinct from $\mathscr{P}(M)$. However, we have

LEMMA 1.2. If $M$ is an $A$-module which is bounded above, then the map $f$ : $\mathscr{P}(M) \rightarrow P \otimes^{\Delta} M$ defined by

$$
f\left(x^{l-1} \otimes m\right)=\sum_{j} x^{l-j-1} \otimes \mathrm{Sq}^{j} m
$$

is an $S$-linear isomorphism with inverse $g$ defined by

$$
g\left(x^{l-1} \otimes m\right)=\sum_{j} x^{l-j-1} \otimes \chi \mathrm{Sq}^{j} m .
$$

In particular, $\mathcal{P}(M)$ is an $A$-module.

Proof. First note that the sums giving $f$ and $g$ are finite since $M$ is bounded above; in fact, for the lemma it suffices to suppose that, for any $m \in M$, Sq $m$ and $\chi \mathrm{Sq} m$ are finite sums. Furthermore $f$ and $g$ are clearly inverse isomorphisms by the definition of the conjugation $\chi$ in the Hopf algebra $S$. The check that $f$ is $S$-linear leads to the identity asserted in the following lemma, which completes the proof.

LEMMA 1.3. In the Steenrod algebra A,

$$
\sum_{i, j \in \mathbf{N}}\left(\begin{array}{c}
l-j-1 \\
k-i
\end{array}\right) \mathrm{Sq}^{i} \mathrm{Sq}^{j}=\sum_{i, j \in \mathbf{N}}\left(\begin{array}{c}
l-j-1 \\
k-2 j
\end{array}\right) \mathrm{Sq}^{i} \mathrm{Sq}^{j}
$$

for any $k \in \mathbf{N}, l \in \mathbf{Z}$.

Proof (N. J. KUHN). With the indicated conventions regarding binomial coefficients, the Adem relation for $\mathrm{Sq}^{i} \mathrm{Sq}^{j}$ is valid for any pair $i, j \in \mathbf{N}$. Thus

$$
\sum_{i, j}\left(\begin{array}{c}
l-j-1 \\
k-i
\end{array}\right) \mathrm{Sq}^{i} \mathrm{Sq}^{j}=\sum_{i, j, n}\left(\begin{array}{c}
l-j-1 \\
k-i
\end{array}\right)\left(\begin{array}{c}
j-n-1 \\
i-2 n
\end{array}\right) \mathrm{Sq}^{i+j-n} \mathrm{Sq}^{n}
$$

Substituting $m=i+j-n$ and $p=i-2 n$, we get

$$
\sum_{m, n, p}\left(\begin{array}{c}
l-m+n-1+p \\
k-2 n-p
\end{array}\right)\left(\begin{array}{c}
m-2 n-1-p \\
p
\end{array}\right) \mathrm{Sq}^{m} \mathrm{Sq}^{n}
$$

Using Adem's relation [1, (25.3)]

$$
\sum_{p}\left(\begin{array}{c}
b+p \\
c-p
\end{array}\right)\left(\begin{array}{c}
a-p \\
p
\end{array}\right)=\left(\begin{array}{c}
a+b+1 \\
c
\end{array}\right)
$$

we get $\sum_{m, n}\left(\begin{array}{c}l-n-1 \\ k-2 n\end{array}\right) \mathrm{Sq}^{m} \mathrm{Sq}^{n}$ as desired. 
Proposition 1.4 (W. M. Singer [12]). $\mathcal{P}$ gives a functor from A-modules to A-modules.

Proof. Let $M$ be an arbitrary $A$-module, and for $d \in \mathbf{Z}$ let $M^{d}$ be the sub$A$-module of elements of degree greater than $d$. Pick $m \in M, l \in \mathbf{Z}$, and $r \in$ $\operatorname{ker}(S \rightarrow A)$, and write $r\left(x^{l-1} \otimes m\right)=\Sigma_{j} x^{j-1} \otimes m_{j}$. Let $d$ be such that $\left|m_{j}\right| \leqslant d$ for all $j$. As $\mathscr{P}$ is exact, we have an exact sequence

$$
0 \rightarrow \mathscr{P}\left(M^{d}\right) \rightarrow \mathscr{P}(M) \rightarrow \mathcal{P}\left(M / M^{d}\right) \rightarrow 0 .
$$

The $A$-module $M / M^{d}$ is bounded above, so by Lemma 1.2,r( $\left.x^{l-1} \otimes m\right)$ maps to 0 there, and hence $\Sigma_{j} x^{j-1} \otimes m_{j} \in \mathcal{P}\left(M^{d}\right)$. It is therefore zero for degree reasons, completing the proof.

Let $\varepsilon: \mathcal{P}(M) \rightarrow M$ by

$$
\varepsilon\left(x^{l-1} \otimes m\right)=\mathrm{Sq}^{l} m \text {. }
$$

This is clearly $A$-linear, and if $M$ is bounded above corresponds via Lemma 1.2 to the map $\varepsilon \otimes 1: P \otimes^{\Delta} M \rightarrow \mathbf{Z}_{2} \otimes^{\Delta} M=M$ where $\varepsilon\left(x^{l-1}\right)=\delta_{l}^{0}$. We remark that W. $H$. Lin has shown [6] that $\varepsilon$ induces an isomorphism in $\operatorname{Tor}_{*}^{A}\left(Z_{2},-\right)$ for $M=Z_{2}$. It follows by the 5-lemma that it is an isomorphism for $M$ finite, or indeed for $M$ a direct limit of finite $A$-modules-e.g. for $M$ bounded above. The author and, independently, J. F. Adams and J. Gunawardena, have recently shown that it is an isomorphism for general $M$.

Let $T(M)$ be the sub- $A$-module of $\mathscr{P}(M)$ generated as a vector space by $\left\{x^{l-1} \otimes m: l \in \mathbf{N}, m \in M\right\}$. Singer's main theorem, which we quote without the proof, is

THEOREM 1.5. $\varepsilon_{*}: \operatorname{Tor}_{s}^{A}\left(\mathbf{Z}_{2}, T(M)\right) \rightarrow \operatorname{Tor}_{s}^{A}\left(\mathbf{Z}_{2}, M\right)$ is trivial for $s>0$.

By duality, we have

THEOREM 1.6. $\varepsilon^{*}: \operatorname{Ext}_{A}^{s}\left(M, \mathbf{Z}_{2}\right) \rightarrow \operatorname{Ext}_{A}^{s}\left(T(M), \mathbf{Z}_{2}\right)$ is trivial for $s>0$.

2. Let $F^{n}$ be the subvector space of $A$ with basis consisting of the admissible squares of length at least $n$.

Lemma 2.1. (a) $F^{n}$ is a left ideal in $A$. (b) If $\theta \in F^{n}$ and $k>|\theta|$, then $\mathrm{Sq}^{k} \theta \in F^{n+1}$.

We postpone the proof of this lemma. Our object in this section is to prove

THEOREM 2.2. For any $n \geqslant 0$, the map $\operatorname{Ext}_{A}^{*}\left(F^{n}, \mathbf{Z}_{2}\right) \rightarrow \operatorname{Ext}_{A}^{*}\left(F^{n+1}, \mathbf{Z}_{2}\right)$ induced by the inclusion $F^{n+1} \rightarrow F^{n}$ is trivial.

For this purpose, we consider the $A$-module map $\alpha: A \rightarrow T(A)$ sending 1 to $x^{-1} \otimes 1$; cf. [12, Proposition 5.4]. Notice that $\alpha$ splits the map $\varepsilon: T(A) \rightarrow A$. Furthermore

Proposition 2.3. $\alpha\left(F^{n+1}\right) \subseteq T\left(F^{n}\right)$ for $n>0$.

Proof. Let $I$ be admissible of length $n$. We claim that

$$
\alpha\left(\mathrm{Sq}^{I}\right)=\sum_{\substack{l(J)>n-1 \\|I| / 2<l<i_{1}}}^{*} x^{l-1} \otimes \mathrm{Sq}^{J}
$$


where the star indicates that some terms have zero coefficient. This is proved by induction on $n$, using Lemma 2.1 and the following easy consequence of (1.1).

(2.5) If $k \geqslant 2 l$, then

$$
\mathrm{Sq}^{k}\left(x^{l-1} \otimes m\right)=\sum_{k / 2+l<j<k}^{*} x^{j-1} \otimes \mathrm{Sq}^{k+l-j} m \text {. }
$$

With $m=1 \in A$ and $l=0$, (2.5) implies $n=1$ of (2.4). Now let $I=\left(i_{1}, I^{\prime}\right)$; by inductive hypothesis

$$
\alpha\left(\mathrm{Sq}^{I^{\prime}}\right)=\sum_{\substack{l(J)>n-2 \\\left|I^{\prime}\right| / 2<l<i_{2}}} * x^{l-1} \otimes \mathrm{Sq}^{J}
$$

Now $\alpha\left(\mathrm{Sq}^{I}\right)=\mathrm{Sq}^{i_{1}} \alpha\left(\mathrm{Sq}^{I^{\prime}}\right)$, so we must consider $\mathrm{Sq}^{i_{1}}\left(x^{l-1} \otimes \mathrm{Sq}^{J}\right)$. Since $i_{1}>2 i_{2}>$ $2 l,(2.5)$ implies

$$
\mathrm{Sq}^{i_{1}}\left(x^{l-1} \otimes \mathrm{Sq}^{J}\right)=\sum_{i_{1} / 2+l<j<i_{1}}^{*} x^{j-1} \otimes \mathrm{Sq}^{i_{1}+l-j} \mathrm{Sq}^{J}
$$

Now in (2.6), $l+|J|=\left|I^{\prime}\right| \leqslant 2 l$, so $l \geqslant|J|$; so we have $i_{1}+l-j>|J|$, and by Lemma 2.1, $\mathrm{Sq}^{i_{1}+l-j} \mathrm{Sq}^{J} \in F^{n-1}$. Also $\frac{1}{2} i_{1}+l \geqslant \frac{1}{2} i_{1}+\frac{1}{2}\left|I^{\prime}\right|=\frac{1}{2}|I|$, completing the induction.

Proof of Theorem 2.2. By Proposition 2.3, the projection $\pi: A / F^{n+1} \rightarrow A / F^{n}$ factors through $\varepsilon: T\left(A / F^{n}\right) \rightarrow A / F^{n}$. According to Theorem $1.6, \pi$ thus induces zero in $\operatorname{Ext}_{A}^{s+1}\left(-, Z_{2}\right)$ for $s \geqslant 0$; but this is just the map induced by $F^{n+1} \rightarrow F^{n}$ in $\operatorname{Ext}_{A}^{s}\left(-, \mathbf{Z}_{2}\right)$, so the result follows.

Proof of Lemma 2.1. Let $(a)_{n}$ and (b) $)_{n}$ denote the two assertions of the lemma. We prove them together, by induction on $n$. For $n=1$, we find that $F^{1}$ is the augmentation ideal, and (b) $)_{1}$ is clear from a glance at the Adem relation involved. Now assume $(\mathrm{a})_{n-1}$ and $(\mathrm{b})_{n-1}$. Let $I$ be admissible of length $l(I)>n$, and write $I=\left(i_{1}, I^{\prime}\right)$. We prove that $\mathrm{Sq}^{k} \mathrm{Sq}^{I} \in F^{n}$. If $k \geqslant 2 i_{1}, \mathrm{Sq}^{k} \mathrm{Sq}^{I} \in F^{n+1}$. Otherwise, the Adem relations give

$$
\mathrm{Sq}^{k} \mathrm{Sq}^{I}=\sum_{0<2 j<k}^{*} \mathrm{Sq}^{k+i_{1}-j} \mathrm{Sq}^{j} \mathrm{Sq}^{I^{\prime}}
$$

where the star indicates that coefficients have been omitted. By $(a)_{n-1}, \mathrm{Sq}^{j} \mathbf{S q}^{I^{\prime}} \in$ $F^{n-1}$. Furthermore, $k+i_{1}-j \geqslant 2 j+i_{1}-j=j+i_{1}>j+\left|I^{\prime}\right|$ by admissibility, so, by (b) $)_{n-1}$, each term lies in $F^{n}$, as desired. For (b) $)_{n}$, note that $i_{1}-1-j>k-$ $2 j$ is necessary for the binomial coefficient to be nonzero: that is, $j \geqslant k-i_{1}$. Thus if $k \geqslant|I|$, then $j \geqslant k-i_{1} \geqslant|I|-i_{1}=\left|I^{\prime}\right|$, so by (b) $)_{n-1}, \mathrm{Sq}^{j} \mathrm{Sq}^{I^{\prime}} \in F^{n}$. The leading index in each term of the admissible expansion of this element is less than $\frac{1}{2}\left(j+\left|I^{\prime}\right|\right)$, since $n>1$. But then $k+i_{1}-j>2 j+\left|I^{\prime}\right|-j=j+\left|I^{\prime}\right|$, so no further Adem relations are needed to make $\mathrm{Sq}^{k+i_{1}-j} \mathrm{Sq}^{j} \mathrm{Sq}^{I^{\prime}}$ admissible, and each term lies in $F^{n+1}$. Q.E.D.

3. We show how Theorem 2.2 implies the Main Theorem.

Let $M=S^{0} \cup_{2} e^{1}$ and let $H_{2}$ be the mod 2 Eilenberg-Mac Lane spectrum. The composite $H \wedge M \rightarrow H_{2} \wedge H_{2} \stackrel{\mu}{\rightarrow} H_{2}$, in which $H \rightarrow H_{2}$ is reduction, $M \rightarrow H_{2}$ the inclusion of the 1-skeleton, and $\mu$ the multiplication, induces an $A$-module isomorphism $\beta: A \rightarrow \bar{A} \otimes^{\Delta} E[e]$. To describe it, let $\Delta_{j}$ denote the "Dirac sequence" with 1 
for the $j$ th entry and 0 elsewhere. Let ${ }^{-}: A \rightarrow \bar{A}$ be the projection. Then for $I$ admissible and of length $n$,

$$
\beta\left(\mathrm{Sq}^{I}\right)=\overline{\mathrm{Sq}^{I}} \otimes 1+\sum_{j=1}^{n} \overline{\mathrm{Sq}^{I-\Delta_{j}}} \otimes e,
$$

by the usual formula for the diagonal $\mu^{*}$ in $A$. We claim that

$$
\beta\left(F^{n}\right) \subseteq \bar{F}^{n-1} \otimes E[e]
$$

that is, $\overline{\mathrm{Sq}^{I-\Delta_{j}}} \in \bar{F}^{n-1}$ for each $j$. If $I-\Delta_{j}$ is admissible, $\mathrm{Sq}^{I-\Delta_{j}} \in F^{n}$ unless $j=n$ and $i_{n}=1$, when $\mathrm{Sq}^{I-\Delta_{j}} \in F^{n-1}$. If not, then $i_{j}=2 i_{j+1}$, so $\mathrm{Sq}^{2 i-1} \mathrm{Sq}^{i}=0$ (with $i=i_{j+1}$ ) occurs in $\mathrm{Sq}^{I-\Delta_{j}}$, and the term is trivial.

We therefore have a map of short exact sequences (which in fact expresses $F^{n}$ as a pull-back)

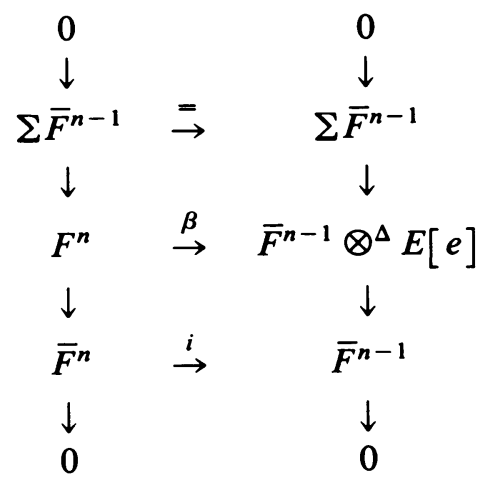

Consequently, the boundary homomorphism $\delta$ associated to the left-hand column factors through $i^{*}$; in fact $\delta x=i^{*}\left(h_{0} x\right)$, where $h_{0}$ is the generator of $\operatorname{Ext}_{A}^{1,1}\left(\mathbf{Z}_{2}, \mathbf{Z}_{2}\right)$.

Now consider the following commutative diagram with exact columns.

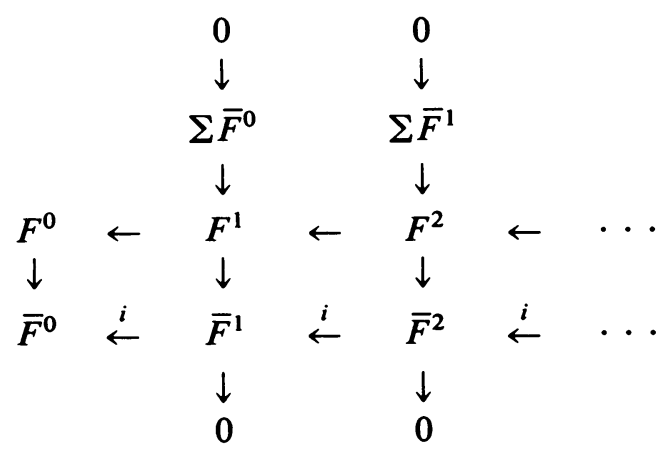

The upper horizontal arrows are zero in Ext by Theorem 2.2. Take $x \in$ $\operatorname{Ext}_{A}^{s}\left(\bar{F}^{n}, \mathbf{Z}_{2}\right)$. If $s<0$, then $i^{*} x=0$ for certain. We proceed by induction on $s . i^{*} x$ pulls back to 0 in $\operatorname{Ext}_{A}^{s}\left(F^{n+1}, \mathbf{Z}_{2}\right)$, so there exists $\bar{x} \in \operatorname{Ext}_{A}^{s-1}\left(\Sigma \bar{F}^{n}, \mathbf{Z}_{2}\right)$ such that $i^{*} x=\delta \bar{x}$, i.e., $i^{*} x=i^{*} h_{0} \bar{x}$. However, $i^{*}\left(h_{0} \bar{x}\right)=h_{0} i^{*}(\bar{x})$, which is zero by inductive assumption. This completes the proof.

REMARK 3.3. It is not hard to show that $\Sigma^{-n} \bar{F}^{n} / \bar{F}^{n+1}$ is an unstable $A$-module; for example, $\Sigma^{-1} \bar{F}^{1} / \bar{F}^{2}=\bar{H}^{*}\left(\mathbf{R} P^{\infty}\right)$. We are led to conjecture that $\Sigma^{-n} \mathrm{Sp}^{2 n} S^{0} / \mathrm{Sp}^{2^{n-1}} S^{0}$ is the suspension spectrum of a space $L(n)$. This also fits well 
with [9]. The proper construction of $L(n)$ might lead to a proof that (if $\Omega^{\infty}$ denotes formation of the 0 -space of the $\Omega$-spectrum) $Q L(n) \rightarrow \Omega^{\infty} \Sigma^{-n} H / \mathrm{Sp}^{2^{n-1}} S^{0}$ splits, generalizing the Kahn-Priddy theorem and proving Whitehead's conjecture.

\section{REFERENCES}

1. J. Adem, The relations on Steenrod powers of cohomology classes, Algebraic Geometry and Topology (R. H. Fox et al., Eds.), Princeton Univ. Press, Princeton, N.J., 1957, pp. 191-242.

2. D. W. Anderson and D. W. Davis, A vanishing line in homological algebra, Comment Math. Helv. 48 (1973), 318-327.

3. A Dold and R. Thom, Quasifaserungen und unendliche symmetriche Produkte, Ann. of Math. (2) 67 (1958), 239-281.

4. I. M. James, E. Thomas, H. Toda and G. W. Whitehead, On the symmetric square of a sphere, J. Math. Mech. 12 (1963), 771-776.

5. D. S. Kahn and S. B. Priddy, The transfer and stable homotopy theory, Math. Proc. Cambridge Philos. Soc. 83 (1978), 103-111.

6. W. H. Lin, D. W. Davis, M. E. Mahowald and J. F. Adams, Calculation of Lin's Ext groups, Math. Proc. Cambridge Philos. Soc. 87 (1980), 459-470.

7. W. H. Lin, Algebraic Kahn-Priddy Theorem, preprint.

8. R. J. Milgram (Ed.), Problems presented to the 1970 AMS summer colloquium in algebraic topology, Algebraic Topology, Proc. Sympos. Pure Math., vol. 22, Amer. Math. Soc., Providence, R.I., 1971, pp. 187-201.

9. H. R. Miller, A spectral sequence for the homology of an infinite delooping, Pacific J. Math. 79 (1978), 139-155.

10. H. R. Miller and C. W. Wilkerson, Vanishing lines for modules over the Steenrod algebra, J. Pure Appl. Algebra (to appear).

11. M. Nakaoka, Cohomology mod p of symmetric products of spheres, J. Inst. Poly. Osaka City Univ. 9 (1958), 1-18.

12. W. M. Singer, An embedding theorem for the homology of the Steenrod algebra (to appear).

13. P. J. Welcher, Symmetric products and the stable Hurewicz homomorphism, Illinois J. Math. (to appear).

14. __ Symmetric fiber spectra and $K(n)$-homology acyclicity, Indiana Univ. Math. J. (to appear).

Department of Mathematics, University of Washington, Seattle, Washington 98195 